\title{
Rituximab-conjugated and doxorubicin-loaded microbubbles combined with ultrasound irradiation inhibits proliferation and induces apoptosis in Raji cell lines
}

\author{
SHOUBING ZHOU ${ }^{1}$, SHIYA ZHENG ${ }^{1}$, YONGFENG SHAN ${ }^{3}$, LULU LI $^{1}$, \\ XIU ZHANG ${ }^{1}$ and CAILIAN WANG ${ }^{1,2}$ \\ ${ }^{1}$ Medical School, Southeast University, Nanjing, Jiangsu 210009; ${ }^{2}$ Department of Oncology, Zhongda Hospital, \\ Medical School, Southeast University, Nanjing, Jiangsu 210009; ${ }^{3}$ Department of Oncology, \\ Traditional Chinese Medicine Hospital, Wuxi, Jiangsu, P.R. China
}

Received August 29, 2015; Accepted October 11, 2015

DOI: 10.3892/or.2015.4468

\begin{abstract}
Doxorubicin (DOX) is one of the most important medicines used for the treatment for B cell lymphoma, yet its clinical efficacy is often limited by severe adverse effects. Drug-loaded microbubbles, combined with ultrasound (US) irradiation, has shown great promise in reducing DOX-induced side effects and improving therapeutic efficacy. Nevertheless, these drug-loaded microbubbles are non-targeted microbubbles with comparatively suboptimal efficiency. Therefore, we synthesized targeted and DOX-loaded microbubbles (DMs), combined with US irradiation, for triggering drug release in lymphoma B cells. DMs were coated with rituximab via a biotin-avidin linkage to target Raji cells that overexpress the CD-20 antigen. In the present study, the cell viability after treatment with rituximab-conjugated DMs (RDMs) containing $0.25,0.5$ and $1.0 \mu \mathrm{g} / \mathrm{ml}$ DOX + US was 45.69 $\pm 6.85,25.31 \pm 2.60$ and $15.67 \pm 2.83 \%$, respectively, which demonstrated that RDMs + US produced significantly higher cytotoxicity than the other treatments. The early apoptosis ratio in the Raji cells at $48 \mathrm{~h}$ after the treatment was $32.4 \pm 2.84 \%$, which was notably higher than the ratio in the other treatment groups. The results confirm the hypothesis that US-mediated targeting of CD-20positive B cell lymphoma and the use of DMs may improve the DOX therapeutic efficiency.
\end{abstract}

\section{Introduction}

B cell non-Hodgkin's lymphoma (NHL) is a high incidence malignancy of lymphoid tissues that affects patients all over the world and accounts for $\sim 85 \%$ of NHL cases (1). Currently, chemotherapy and radiotherapy, particularly the former, are

Correspondence to: Professor Cailian Wang, Department of Oncology, Zhongda Hospital, Medical School of Southeast University, 87 Dingjiaqiao Road, Nanjing, Jiangsu 210009, P.R. China

E-mail: wangcailianseu@yahoo.com

Key words: B cell lymphoma, ultrasound, apoptosis, doxorubicin, microbubbles the main approaches for the treatment of B cell NHL in clinical applications. At present, rituximab plus cyclophosphamide, doxorubicin (DOX), vincristine, and prednisone (R-CHOP) has become the first-line treatment regimen for B cell NHL. The 5-year overall survival rate of patients with B cell NHL has improved to $\sim 60 \%$ due to the application of $\mathrm{R}-\mathrm{CHOP}$ regimens (2). Unfortunately, DOX, as the major cytotoxic agent of $\mathrm{R}-\mathrm{CHOP}$, is often limited in clinical application due to severe adverse effects such as myelosuppression, cardiomyopathy and congestive heart failure (3), which are mainly associated with non-specific biodistribution of DOX. Therefore, a novel strategy to improve the targeting of DOX to B cell NHL is urgently needed for minimizing the side effects and maximizing the therapeutic efficacy.

The appearance of targeted drug delivery systems based on a combination of polymeric microbubbles (MBs) and ultrasound (US) provides a promising approach for improving the targeting of traditional chemotherapeutic drugs. Additionally, compared with other drug delivery systems, targeted drug delivery systems have the advantages of satisfactory chemical stability, biodegradability, low toxicity and easy chemical modification (4-6). Previous research has confirmed that carriers conjugated to monoclonal antibodies (7-9), galactoseconjugated chitosan $(10)$, transferrin $(11,12)$, peptides $(13,14)$, and folate $(15,16)$ for drug delivery have produced promising therapeutic effects in solid tumors. A non-targeted drug delivery carrier called DOX-loaded MBs (DMs) was successfully synthesized, as described previously (17). However, DMs easily aggregate in the reticuloendothelial system, leading to a low drug concentration at the tumor site. To improve DOX targeting, enhance therapeutic efficacy, and reduce systemic toxicity, rituximab-conjugated DMs (RDMs) were developed. Rituximab, a monoclonal antibody, is primarily used for immunotherapy of CD-20-positive B cell lymphoma (18). However, in those drug delivery system formulations, rituximab is intended to serve as a targeted ligand rather than as a therapeutic agent.

In the present study, rituximab was conjugated onto the surface of DMs, and US was used to destroy RDMs to increase the release of DOX and improve the targeting and cytotoxicity 
of DOX against CD-20-positive human lymphoma Raji cells. The physicochemical properties and therapeutic efficacy of the RDMs were also evaluated.

\section{Materials and methods}

\section{Materials and preparation}

Materials. Maleic anhydride grafting poly-L-lactic acid (MPLA, MW=10,000 Da) was purchased from Shandong Shu YuanBiotechnology Co.,Ltd.Poly(vinylalcohol)(PVA,87-89\%, MW=31,000-50,000) was obtained from Sigma-Aldrich Co. DOX was obtained from Shenzhen Wanle Pharmaceutical Co., Ltd. Amine-Peg2000-Biotin, the EZ-Link ${ }^{\mathrm{TM}}$ Sulfo-LCBiotinylation kit and 1-ethyl-3-(3-dimethylaminopropyl) carbodiimide hydrochloride (EDC) were purchased from Thermo Fisher Scientific. Rituximab was obtained from Hoffmann-La Roche, Ltd. The Annexin V-FITC cell apoptosis detection kit, DAPI (4,6-diamidino-2-phenylindole) staining solution and Cell Counting Kit-8 were purchased from Beyotime Biotechnology Co., Ltd. Monoclonal antibodies, including bcl-2, bax, caspase-3 were supplied by Santa Cruz Biotechnology Co., Ltd. All reagents were of analytical grade.

Preparation of the DMs. Polymer MBs encapsulating DOX were prepared by a double US emulsion (water/oil/water) evaporation process. MPLA ( $0.5 \mathrm{~g})$ was completely dissolved in $10 \mathrm{ml}$ of chloroform liquid with stirring. The solution was then mixed with $5 \mathrm{mg}$ DOX (dissolved in $1.5 \mathrm{ml}$ distilled water) and the mixture was emulsified by a US emulsification apparatus for $90 \mathrm{~min}$ at $100 \mathrm{~W}$. The mixture was then mixed with $0.5 \mathrm{ml}$ Span-80. After that, the vial was degassed and reperfused with nitrogen with stirring $(23,000 \mathrm{rpm}, 5 \mathrm{~min})$ to form a primary uniform and stable emulsified microbubble solution. The primary emulsion was poured into the cold PVA $(50 \mathrm{ml}, 5 \%)$ solution containing Tween-80, and stirred at $21,000 \mathrm{rpm}$ at room temperature for $0.5 \mathrm{~h}$ for the second emulsion. The double emulsion was poured into $50 \mathrm{ml}$ of an isopropyl alcohol solution $(2.5 \%, \mathrm{w} / \mathrm{v})$ and then the final solution was mechanically stirred at room temperature for $3 \mathrm{~h}$ to volatilize the chloroform liquid. The supernatant was discarded after the solution was centrifuged at 4,800 rpm for $5 \mathrm{~min}$. Then, the remaining supernatant was removed after centrifugation at $1,800 \mathrm{rpm}$ for $5 \mathrm{~min}$; the solution was resuspended with distilled water and washed with ultrapure water several times until the supernatant became transparent. The precipitates resuspended in ultrapure water were preserved in a $4^{\circ} \mathrm{C}$ refrigerator. Pure MBs were prepared similarly but without DOX solution. MBs were sterilized by cobalt $60\left({ }^{60} \mathrm{Co}\right)$ irradiation.

Preparation of the RDMs. Covalent binding of the activated carboxyl groups on the surface of DMs was accomplished via the EDC chemistry method in the presence of N-hydroxysuccinimide (NHS). The prepared DMs were dissolved in PBS buffer ( $\mathrm{pH}=4.7)$, and EDC and NHS, in an equimolar ratio, were added into the DM solution. The carboxyl groups were activated for $1 \mathrm{~h}$ at room temperature by stirring. The supernatant was discarded after the mixture was centrifuged. Then the precipitations were suspended in PBS buffer, $\mathrm{pH}=7.4$ ), and Amine-Peg2000-Biotin was added to the MES buffer. Subsequently, the mixture was allowed to react for $2 \mathrm{~h}$ at room temperature with stirring to obtain the biotinylated
DMs. For the apoptosis experiment, the biotinylated DMs were incubated with DyLight 488-labeled avidin/avidin without fluorescence (1 mg/ml; Boster, Wuhan, China) for $10 \mathrm{~min}$ at room temperature. The DMs were subsequently centrifuged three times and resuspended with fresh PBS buffer to remove surplus avidin. Thereafter, rituximab was biotinylated using the EZ-Link ${ }^{\mathrm{TM}}$ Sulfo-LC-Biotinylation kit according to the manufacturer's instructions. Finally, biotinylated rituximab was added to the DM suspension and incubated for $10 \mathrm{~min}$. The RDMs were rinsed three times and centrifuged to remove surplus biotinylated rituximab.

\section{Characterization of the RDMs}

Size distribution, zeta potential and assessment of conjugation efficiency. The morphological characterization of the RDMs was investigated using a scanning electron microscope (SEM, Hitachi S-4800; Japan); the structures of the RDMs were analyzed using transmission electron microscopy (TEM, Hitachi H-7600; Japan); and the mean diameter, size distribution and zeta potential of the RDMs were evaluated using a Malvern Zetasizer Nano ZS unit (Malvern Instruments, UK).

The rituximab coupling efficiency was determined by measuring the absorbance of the DyLight 488-avidin solution and biotinylated DMs with a fluorescence spectrophotometer at a maximum excitation wavelength of $493 \mathrm{~nm}$ and a maximum emission wavelength of $518 \mathrm{~nm}$. The quantities of rituximab on the biotinylated DMs [binding efficiency (\%)] were calculated as the ratio of intensity of biotinylated DMs to the intensity of the DyLight 488-labeled avidin samples.

Drug loading efficiency and US-mediated drug release assay. The drug encapsulation efficiency was estimated using ultraviolet-visible spectrophotometry by estimating the amount of encapsulated DOX. The standard curve for the doxorubicin solution was evaluated, and then a sample was centrifuged to collect the precipitate. The MB precipitate was completely solubilized by the same volume of $5 \%$ hydrochloric acid:ethanol solution, and the supernatant was used to determine the OD value at an excitation wavelength of $495 \mathrm{~nm}$. Drug encapsulation efficiency and loading efficiency were calculated using the following equations:

Encapsulation efficiency $=\mathrm{Wa} / \mathrm{Wb} \times 100 \%$; Loading efficiency $=\mathrm{Wa} / \mathrm{Wc} \times 100 \%$;

where Wa represents the total drug amount in the DMs; $\mathrm{Wb}$ represents the total weight of DOX used in the fabrication of the DMs; and Wc represents the total weight of MPLA used in the fabrication of the DMs. Each process was performed in triplicate.

Sonication investigation was performed to evaluate the DOX release response in the sound field. Immediately after the MBs were fabricated, they were used for the ultrasonically triggered release response. The experimental parameters for sonication were similar to those in the literature $(19,20)$. The MBs were enclosed in dialysis bags (MWCO, 10,000 Da), which were placed in a container of $50 \mathrm{ml}$ PBS with shaking at $100 \mathrm{rpm}$ at $37^{\circ} \mathrm{C}$. The MB solution was then sonicated with US (power density, $2.0 \mathrm{~W} / \mathrm{cm}^{2}$; frequency, $1 \mathrm{MHz}$; duty cycle, $50 \%$ ) for $40 \mathrm{sec}$. At the proper intervals, $1 \mathrm{ml}$ of dialysate was removed from the sample and stored at $4^{\circ} \mathrm{C}$ for analysis. At the same time, the same volume of PBS was added to the container 


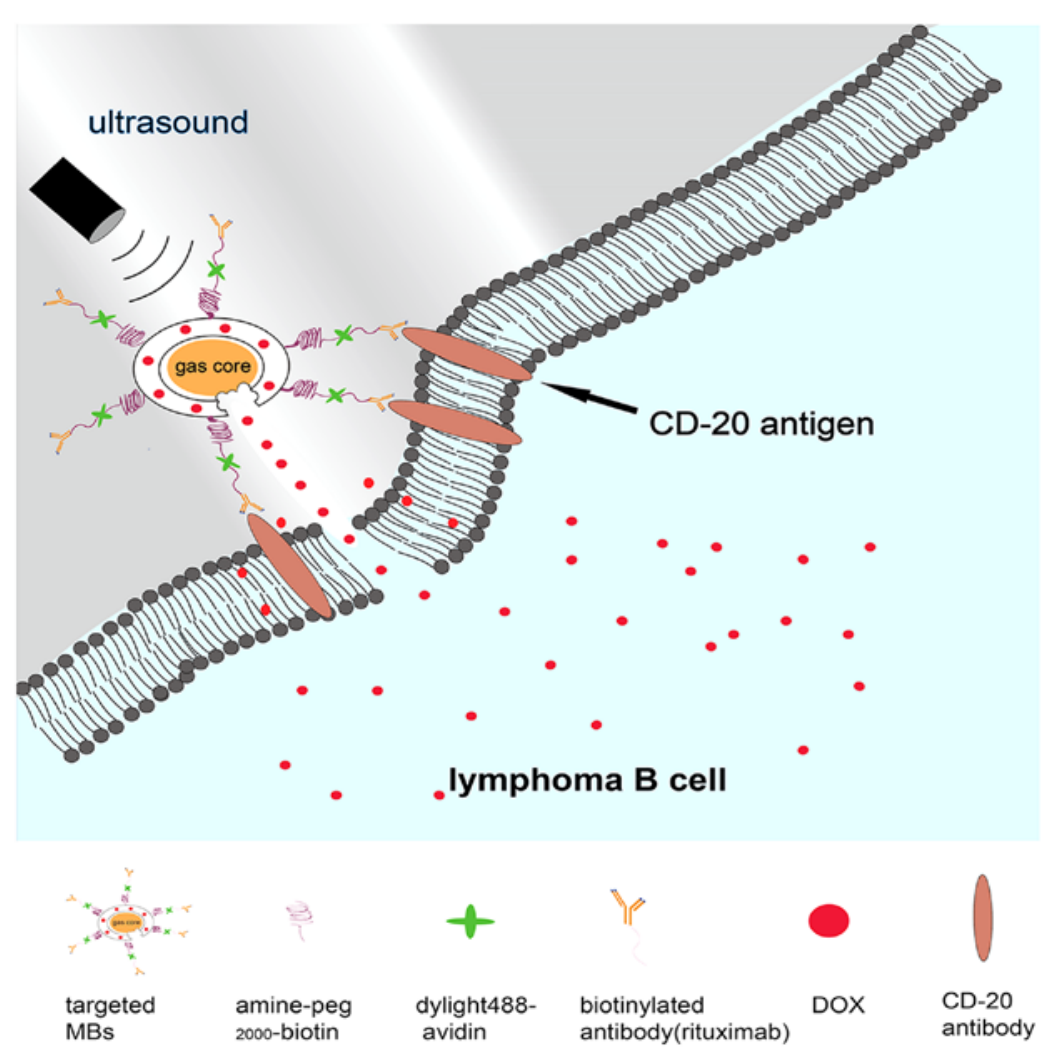

Figure 1. Schematic illustration of the structure of RDMs and the concept of antibody-based and tumor cell targeting-guided drug delivery. After adhesion of targeted doxorubicin-loaded microbubbles and Raji cells via a specific antibody, the encapsulated doxorubicin, triggered by ultrasound, is delivered into the Raji cell.

to ensure a constant volume. The concentration of DOX in the sample was determined using UV-VIS spectrophotometry. Subsequently, the cumulative ratios of DOX release were computed as a function of time. The control group received the same method, except without sonication.

Cell culture and assessment of the target binding. The human lymphoma cell lines, Raji and Jurkat, which were purchased from the Cell Bank of the Chinese Academy of Sciences (Shanghai, China), were grown in RPMI-1640 medium with $10 \%$ (v/v) fetal bovine serum (FBS; Gibco, Australian origin) and $1 \%$ penicillin-streptomycin and incubated in a humidified atmosphere at $5 \% \mathrm{CO}_{2}$ and $37^{\circ} \mathrm{C}$. The Raji cell line was used as a CD-20-positive cell line and the Jurkat cell line was used as a CD-20-negative cell line. For all experiments, the cells growing in suspension were subcultured by centrifugation using a ratio of 1:4.

After the cells were fixed in NEST glass culture dishes, $50 \mu \mathrm{l}$ of targeting RDMs and non-targeting DMs were added into the dishes for the cells to be in contact with the RDMs or DMs by shaking. After $30 \mathrm{~min}$ of target binding at room temperature, the dishes were washed twice with PBS and examined using a confocal laser scanning microscope (CLSM FV1000; Olympus, Japan) using a 100X oil objective at excitation wavelengths of 488 and $585 \mathrm{~nm}$. Blocking tests were performed by pre-incubating the Raji cells with rituximab for $30 \mathrm{~min}$ followed by washing and the targeted RDM interaction. Jurkat cells were used as a control, and the targeting binding of RDMs to Jurkat cells was performed using the same methods.

\section{Evaluation of cell assays in vitro}

Cytotoxicity assay. Raji cells were seeded in 24-well plates at a density of $1 \times 10^{5}$ cells/well in RPMI-1640 medium. The Raji cells were treated with unloaded MBs, DOX, DOX + US, DMs, DMs + US, RDMs and RDMs + US at final DOX concentrations of $0.25,0.5$ and $1.0 \mu \mathrm{g} / \mathrm{ml}$. Wells treated with RPMI-1640 medium were used as controls. MBs were administered to the cells for a 30-min targeted conjugation followed by the application of US as before. Cells were incubated in a humidified atmosphere at $5 \% \mathrm{CO}_{2}$ and $37^{\circ} \mathrm{C}$. After a 48 -h treatment, the cells were washed in PBS three times and replaced with fresh culture medium. Cell viability was detected by measuring the absorbance value at $450 \mathrm{~nm}$ using a microplate reader, according to the instructions provided in the Cell Counting Kit-8.

Cell apoptosis assay. Early apoptosis was determined by evaluating cells with an Annexin V apoptosis kit by flow cytometry using CellQuest software. The cells, after treatment for $48 \mathrm{~h}$, were rinsed with cold PBS twice and resuspended in $195 \mu \mathrm{l}$ of binding buffer solution and stained with $5 \mu \mathrm{l}$ of FITC-labeled Annexin V for 15-20 min at room temperature in the dark. The cells without treatment were considered the control group.

Western blot analysis. After the relevant treatment, the cells were lysed in RIPA buffer containing Protease Inhibitor Cocktail Set I and centrifuged at 12,000 rpm for $10 \mathrm{~min}$ at $4^{\circ} \mathrm{C}$. Protein extraction was performed on ice, resolved by $10 \%$ sodium dodecyl sulfate-polyacrylamide gel electrophoresis (SDS-PAGE) and transferred onto nitrocellulose membranes. 

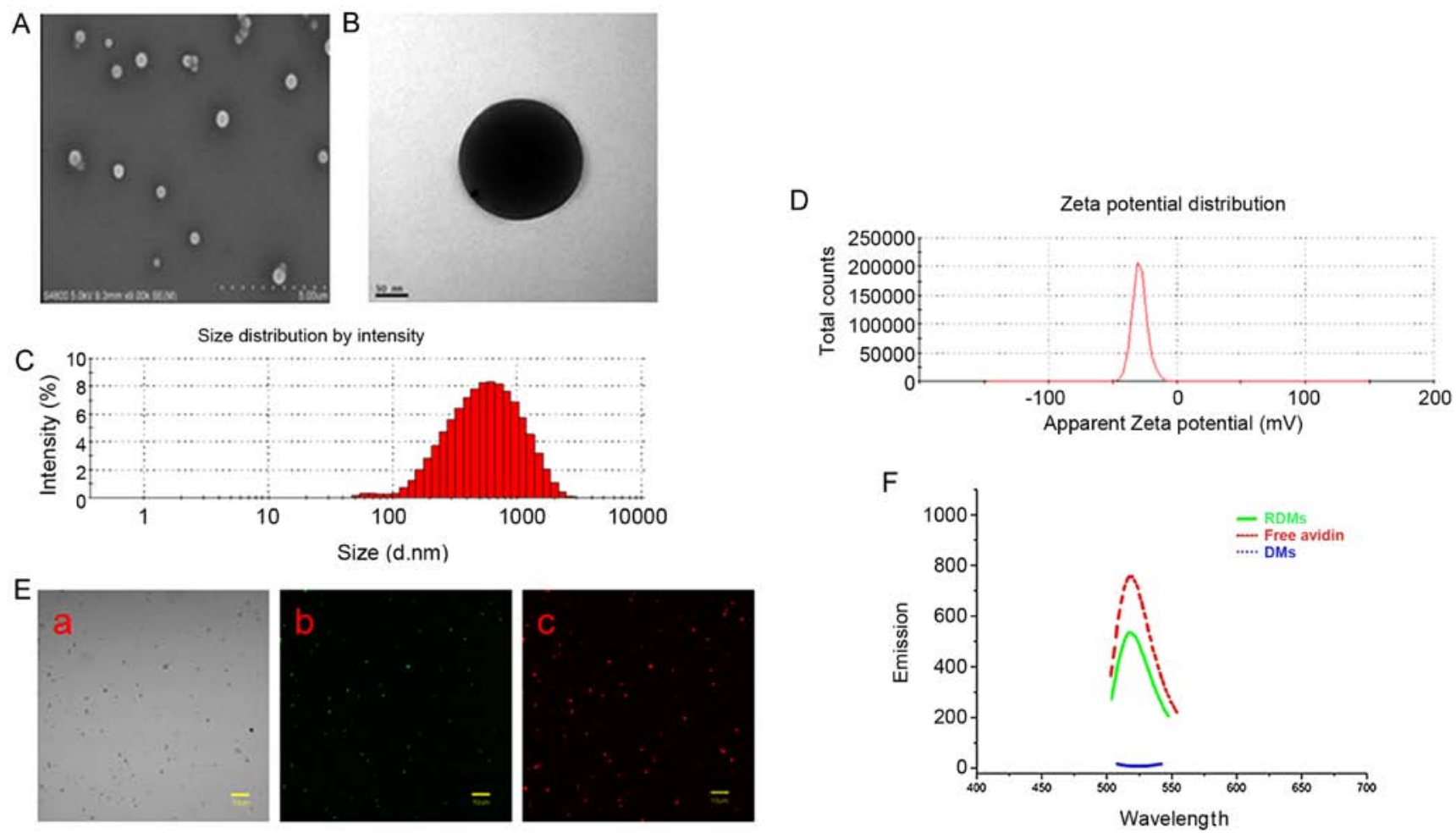

Figure 2. (A) Scanning electron microscopic image of RDMs. (B) Transmission electron microscopic image of RDMs. (C) Size distribution of RDMs by dynamic light scattering (DLS) measurement. (D) Zeta potential of RDMs by DLS measurement. (E) Imaging of RDMs was performed by confocal laser scanning microscopy: (a) bright field, (b) DyLight 488-avidin of DMs (green fluorescence), (c) the encapsulated DOX of DMs (red fluorescence). (F) The fluorescence absorbance of the RDMs, DMs and free avidin were examined to detect the rituximab conjugation efficiency. RDMs, rituximab-conjugated DMs.

Subsequently, the membranes were blocked using $5 \%$ skim milk for $2 \mathrm{~h}$ at room temperature and incubated with bcl-2, bax, caspase- 3 and $\beta$-actin primary monoclonal antibodies overnight at $4^{\circ} \mathrm{C}$ and then with the horseradish peroxidaseconjugated goat anti-rabbit secondary antibody for $2 \mathrm{~h}$ at room temperature. The protein bands were explored by an enhanced chemiluminescence (ECL system; Amersham, UK) detection system and quantified using the Image J software.

Statistical analysis. All experiments were performed in triplicate. Data are presented as the mean \pm standard deviation and were analyzed using SPSS 16.0 software (SPSS, Chicago, IL, USA). Statistical analysis was performed using the Student's t-test. A probability value of $\mathrm{P}<0.05$ was accepted as a significant difference.

\section{Results}

Characterization of RDMs. Fig. 1 illustrates the concept for RDM-targeted Raji cells and controlled release triggered by additional US irradiation. The DOX triggered by sonication was delivered into the Raji cells and enhanced intracellular drug accumulation. The well global morphology and size distribution of the RDMs were distinctly observed in the scanning electron microscopic image (Fig. 2A) and the transmission electron microscopic image (Fig. 2B). The mean diameter of the RDMs was in the range of $505.7 \pm 229.1 \mathrm{~nm}$ (standard deviation) (Fig. 2C). The zeta potential of the RDMs was $-28.9 \pm 11.5 \mathrm{mV}$ (standard deviation) (Fig. 2D and Table I). These findings indicate that the encapsulation of DOX in the
Table I. Characterization of the MBs.

\begin{tabular}{lcc}
\hline Groups & $\begin{array}{c}\text { Size distribution } \\
(\mathrm{nm})\end{array}$ & $\begin{array}{c}\text { Zeta potential } \\
(\mathrm{mV})\end{array}$ \\
\hline Unload MBs & $356.3 \pm 111.7$ & $-22.3 \pm 6.41$ \\
DMs & $407.2 \pm 135.9$ & $-26.7 \pm 5.29$ \\
Rituximab-conjugated DMs & $505.7 \pm 229.1$ & $-28.9 \pm 11.5$ \\
\hline
\end{tabular}

All data were recorded by dynamic light scattering (DLS). Values are means \pm SD $(n=3)$. MBs, microbubbles; DMs, DOX-loaded MBs.

core and binding of rituximab on the surface of DMs slightly affected the particle size and surface charge of the RDMs. Fluorescence imaging of the RDMs demonstrated a dense layer of green (DyLight 488-labeled avidin) and red (DOX auto-fluorescence) fluorescence with morphologies consistent with that observed using bright field CLSM (Fig. 2E). The quantities of the targeting moiety on the surface of the RDMs were evaluated by detecting the fluorescence intensity of the RDM suspensions after the conjugation procedures were performed. The fluorescence intensity of the RDMs was $70.76 \%$, of the DyLight 488-labeled avidin samples, revealing perfect conjugation efficiency of DyLight 488-labeled avidin to biotinylated DMs (Fig. 2F). Due to the extraordinarily high affinity of avidin for biotin, we indirectly presumed the same high adhesion of biotinylated rituximab to the avidinconjugated DMs. 


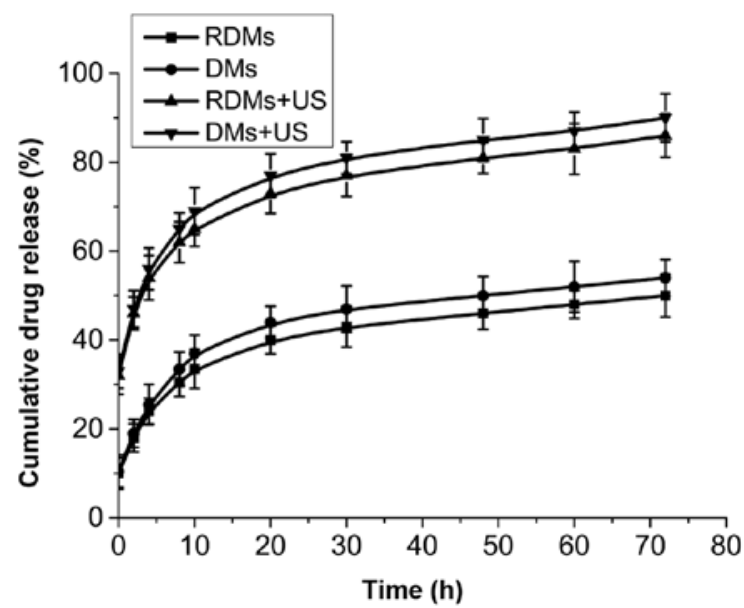

Figure 3. In vitro release of doxorubicin (DOX) from the DMs and RDMs with or without US. DMs, DOX-loaded MBs; RDMs, rituximab-conjugated DMs; US, ultrasound.

DOX-loaded and US-mediated drug release in vitro. The properties of the DMs as a drug delivery system were assessed by the DOX encapsulation efficiency and loading efficiency. The encapsulation and the loading efficiency of DOX in the DMs were $51.2 \pm 1.05$ and $5.07 \pm 0.22 \%$, respectively. In contrast, the encapsulation efficiency of DOX was $49.12 \pm 2.26 \%$ and the loading efficiency was $4.86 \pm 0.31 \%$ in the RDMs. The conjugation of rituximab onto the shell of the DMs did not change the DOX encapsulation efficiency nor the loading efficiency.
The release profiles of DOX from the DMs with/without US irradiation were examined to assess the effects of sonication on DOX release. The release profile was described by the percentage of cumulative release of DOX as a function of time. As shown in Fig. 3, the amount of DOX released from the DMs or RDMs was significantly different between the DMs + US group and the DMs without US group. The results indicated that half of the DOX was unloaded from the DM group with sonication after $5 \mathrm{~h}$ and $\sim 90 \%$ of DOX was discharged after $72 \mathrm{~h}$ post-irradiation. Nevertheless, only $<50 \%$ of the encapsulated DOX was released from DMs without sonication at the same time point of $72 \mathrm{~h}$. Similar experimental findings were also examined between the RDMs + US group and the RDMs without US group. These results implied that US irradiation can promote the release of DOX from DMs or RDMs in a controlled manner due to a cavitation effect.

Targeted properties of RDMs in vitro. To assess the targeting efficiency of RDMs, the affinity of RDMs to Raji cells was determined in vitro. The attachment of RDMs to the Raji cells was significantly higher than the attachment of DMs to the Raji cells. Rituximab enhanced the targeting ability of DMs attachment to the Raji cells. It was distinctly observed, using CLSM, that large amounts of RDMs (green fluorescent and red fluorescent) were present on the membranes of the Raji cells. A few DMs (green fluorescent and red fluorescent) were observed on the membranes of the Raji cells due to the absence of the targeted monoclonal antibody on the surface. The competitive experiment revealed that RDMs were short of the

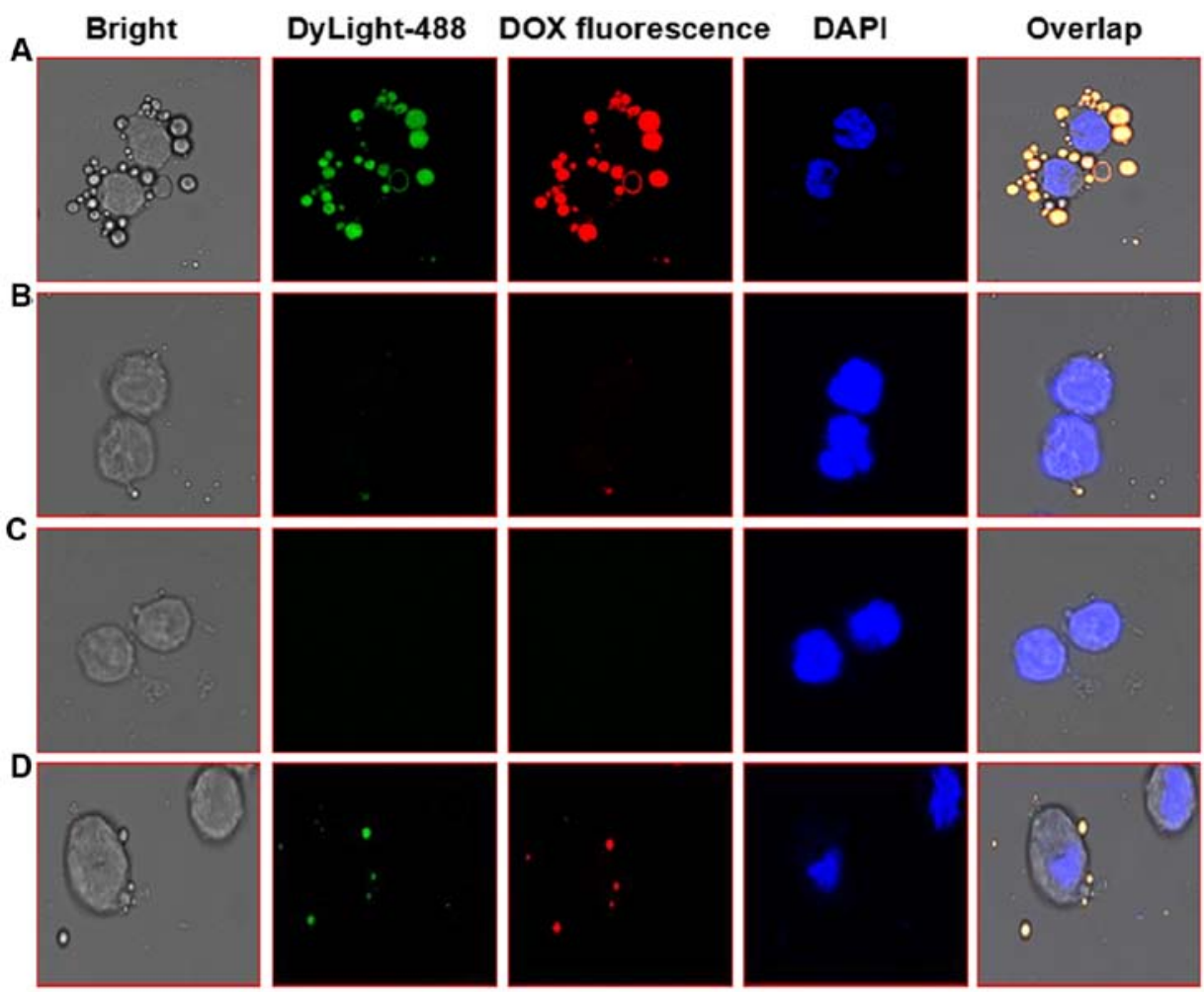

Figure 4. In vitro targeting capacity of RDMs to Raji cells (x100 magnification). Images of the Raji cells after treatment. To observe the conjugational location of RDMs or DMs (green fluorescence or red fluorescence), the cell nuclei were stained with DAPI (blue fluorescence). (A) Raji cells were targeted by RDMs. (B) Raji cells were incubated with DMs. (C) Raji cells were blocked with excess rituximab (1 mg/ml) for $2 \mathrm{~h} \mathrm{before} \mathrm{the} \mathrm{interaction} \mathrm{of} \mathrm{RDMs} \mathrm{and} \mathrm{cells.}$ (D) Jurkat cells were incubated with RDMs. DMs, DOX-loaded MBs; RDMs, rituximab-conjugated DMs. 


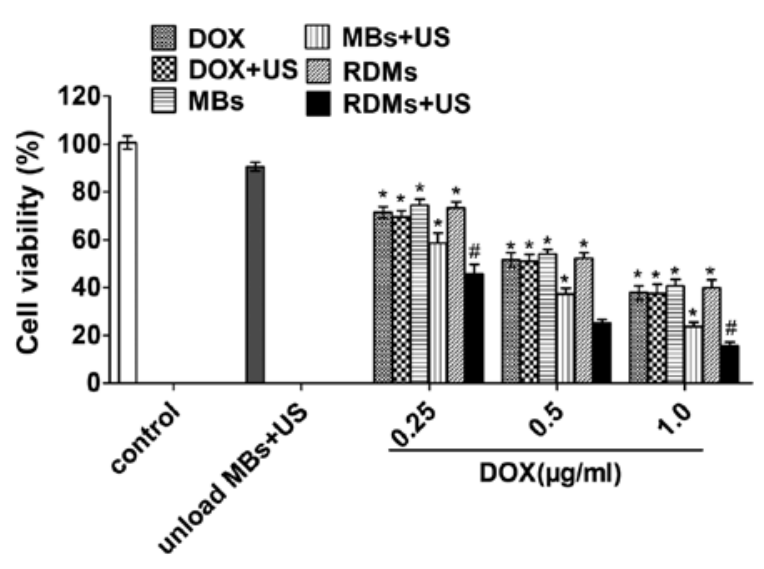

Figure 5. Cell viability was determined after treatment with various regimens containing DOX concentrations of $0.25,0.5$, or $1.0 \mu \mathrm{g} / \mathrm{ml}$ by CCK-8. Data are represented as the mean $\pm \mathrm{SD}(\mathrm{n}=3) .{ }^{*} \mathrm{P}<0.05$ compared with the RDMs + US group; ${ }^{P} \mathrm{P}<0.05$ compared with the RDMs + US group containing $0.5 \mu \mathrm{g} / \mathrm{ml}$ DOX.

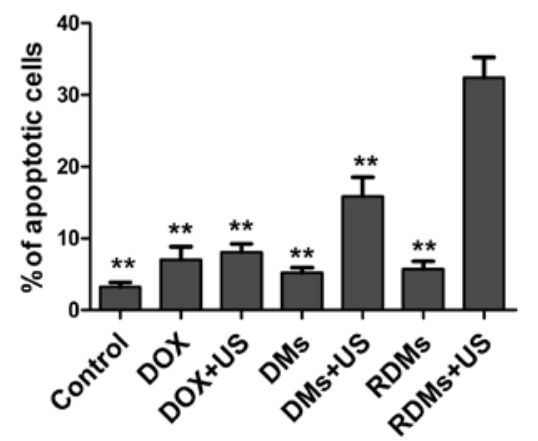

Figure 6. Early apoptotic rates of Raji cells after treatment with various regimens were determined at $48 \mathrm{~h}$ by FCM. Data are shown as the mean $\pm \mathrm{SD}$, ${ }^{* *} \mathrm{P}<0.01$ compared with the RDMs + US group.

targeted guiding ability toward Raji cells. This effect resulted from blockage of the CD20 antigen by pre-incubation with excess rituximab, as indicated by the absence of red and green fluorescence. Furthermore, sparse RDMs (green fluorescent and red fluorescent) were observed on the membranes of the Jurkat cells due to the lack of CD-20 antigen on the surface of Jurkat cells (Fig. 4). These findings suggest the high targeting capacity of RDMs to Raji cells.

Evaluation of cell viability. To evaluate therapeutic potential, the cytotoxic activity of DOX, DOX + US, DMs, DMs + US, RDMs and RDMs + US containing $0.25,0.5$ or $1.0 \mu \mathrm{g} / \mathrm{ml}$ DOX for $48 \mathrm{~h}$ was assessed in cell culture and compared with the control group. As shown in Fig. 5, the cell viability was not altered in the DOX group, DOX + US group, DM group, and RDM group. In contrast, the cell viability, following treatment with DMs containing $0.25,0.5$ or $1.0 \mu \mathrm{g} / \mathrm{ml}$ DOX + US was $58.59 \pm 7.37,37.40 \pm 4.09$ and $23.73 \pm 3.36 \%$, respectively. This percentage changed to $45.69 \pm 6.85,25.31 \pm 2.60$ and $15.67 \pm 2.83 \%$ for the RDMs + US groups. Clearly, the RDMs + US group demonstrated much higher cytotoxicity than the other treatment groups $(\mathrm{P}<0.05$, Fig. 5$)$, demonstrating that RDMs + US significantly inhibit cell proliferation. Treatment with unloaded MBs + US resulted in no obvious
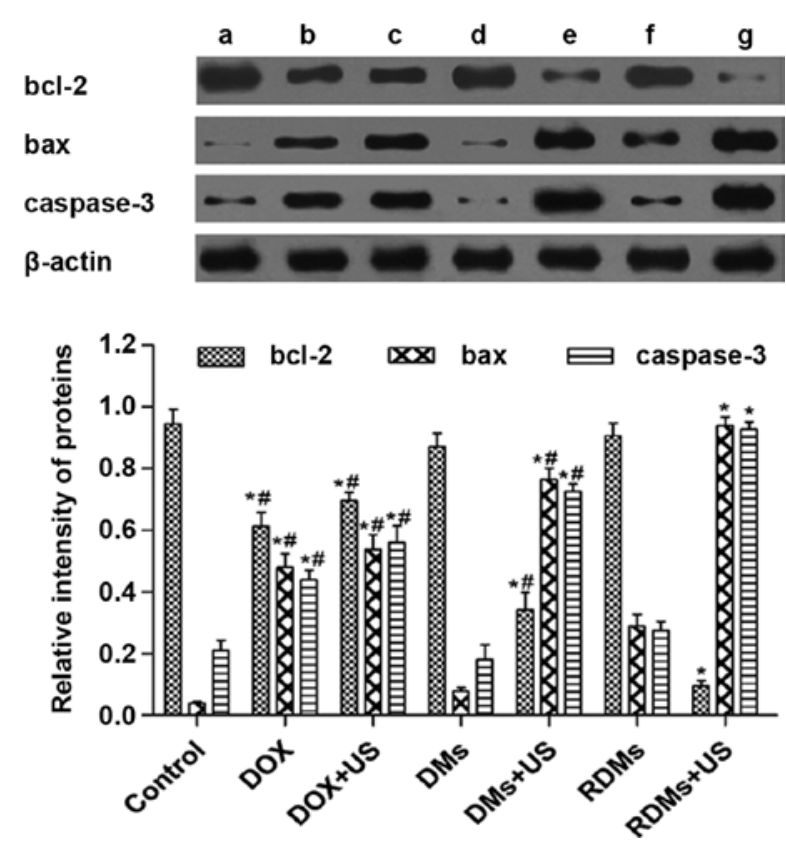

Figure 7. Expression of bcl-2, bax, and caspase-3 protein after different treatments for $48 \mathrm{~h}$. Lane a, control; lane b, DOX; lane c, DOX + US; lane d, DMs; lane e, DMs + US; lane f, RDMs; lane g, RDMs + US. ${ }^{*} \mathrm{P}<0.05$ compared with the control group; ${ }^{\#} \mathrm{P}<0.05$ compared with the RDMs + US group.

cytotoxic effects on the Raji cells compared with the control group ( $\mathrm{P}>0.05$, Fig. 5).

Cellular apoptosis. The early apoptosis efficiencies of Raji cells were evaluated quantitatively by flow cytometry. The early apoptosis efficiencies of the cells treated with the control, DOX, DOX + US, DMs, DMs + US, RDMs and RDMs + US for $48 \mathrm{~h}$ were $3.26 \pm 0.59,7.06 \pm 1.79,8.07 \pm 1.17,5.22 \pm 0.72$, $15.85 \pm 2.68,5.73 \pm 1.10$ and $32.4 \pm 2.84 \%$, respectively. There was no significant difference among the DM, RDM and control groups ( $P>0.05$, Fig. 6). In addition, the DOX and DOX + US groups exhibited moderately increased apoptotic efficiencies compared with the control group ( $\mathrm{P}<0.05$, Fig. 6). However, in comparison with other treatment groups, the DMs + US group and the RDMs + US group resulted in significantly higher apoptosis efficiencies ( $\mathrm{P}<0.01$, Fig. 6). Importantly, the RDMs + US groups showed the highest apoptosis efficiencies than the other treatment groups $(\mathrm{P}<0.01$, Fig. 6).

Expression of Bcl-2, Bax and caspase-3 proteins. As shown in Fig. 7, the bcl-2 protein was overexpressed in the Raji cell lines, whereas bax and caspase- 3 were underexpressed. The protein expression in the Raji cells treated with DMs + US and RDMs + US for $48 \mathrm{~h}$ was notably regulated compared with the control, DOX, DOX + US, DM and RDM groups $(\mathrm{P}<0.05$, Fig. 7). The modulation of these proteins, compared with the control groups, was enhanced in the RDMs + US group. The downregulation rate of bcl-2 protein expression was $1050.28 \pm 30.41 \%$, and the upregulation rates of bax and caspase-3 protein expression were $3134.07 \pm 712.31$ and $440.11 \pm 25.05 \%$. In the DMs + US groups, the corresponding rates were $296.57 \pm 16.05,1686.50 \pm 251.47$ and $397.83 \pm 41.72 \%$ ( $\mathrm{P}<0.05$, Fig. 7). Furthermore, the bcl- 2 and caspase-3 protein expression levels in the Raji cells from the 
DM and RDM groups were not significantly altered relative to the control group ( $\mathrm{P}>0.05$, Fig. 7).

\section{Discussion}

The development of a targeted drug delivery system for MBs, coupled with an antibody that targets the corresponding antigen overexpressed on tumor cells, provides a promising strategy for reducing the severe adverse effects associated with chemotherapeutic drugs (21). Since rituximab is an effective antibody that can specifically interact with the CD20 antigen overexpressed on the extracellular membranes of many human lymphoma B cells, it was used to decorate DMs in our study. Eventually, a targeted drug delivery system with the advantages of rituximab-targeted tumor site orientation and controlled drug release mediated by US, was successfully prepared to enhance the DOX chemotherapeutic efficacy against B cell NHL.

In the present study, rituximab was conjugated onto the surface of DMs through avidin-biotin bridging, and polyethylene glycol (PEG) with hydrophility and perfect biocompatibility was also used to modify the RDMs. At present, the interaction between avidin and biotin is considered to be the non-covalent binding of the highest affinity that is not affected by organic solvents such as reagent concentration, $\mathrm{pH}$, or protein denaturing agent. Furthermore, this linkage has higher stability and specificity compared with other approaches, such as covalent linkage and electrostatic adsorption. Although this linkage approach was successfully confirmed in our experiment, its feasibility must be investigated further due to the unwanted immunogenicity. The RDMs decorated by PEG could decrease nonspecific adsorption of plasma proteins to them, which could enhance recognition and elimination by the reticuloendothelial system before they reach the desired target sites, and increase drug accumulation at the target site (22). To ensure that RDMs easily pass through the tumor neovasculature, the diameter of the RDMs was controlled at approximately $500 \mathrm{~nm}$. Previous studies have confirmed that vascular permeability is notably improved due to expansion of the intercellular space and the lack of basement membrane and smooth muscle. This results in a maximum pore size of approximately 380-780 nm, which allows MBs with a diameter of $<700 \mathrm{~nm}$ to easily pass through the tumor neovasculature (9). Compared with lipid microbubbles, the RDMs established showed great advantages of stability in our work. The RDMs were able to acquire a drug encapsulation efficiency of $49.12 \%$ and a loading efficiency of $4.86 \%$, similar to a previous study (17). Furthermore, the results of the US-mediated drug release assay implied that US can induce MBs to release DOX in a controlled manner, probably due to cavitation effects.

In the present study, RDMs could attach onto the membranes of Raji cells, and comparatively, almost no red DMs surrounded the Raji cells due to the absence of rituximab on the surface. The competitive experiment indicated that RDMs lost their targeting abilities toward Raji cells when the CD-20 antigen was blocked by rituximab in advance. These findings provide direct evidence that RDMs target to Raji cells in vitro. Next, the cytotoxic effect of RDMs to Raji cells was also explored. It showed that the most profound cytotoxic effects after treatment with RDMs containing $0.25,0.5$ or $1.0 \mu \mathrm{g} / \mathrm{ml} \mathrm{DOX}+\mathrm{US}$, were detected, compared with the other treatment groups, respectively. In the present study, the US frequency of $1 \mathrm{MHz}$ was used on the cells at a power intensity of $2.0 \mathrm{~W} / \mathrm{cm}^{2}$ for $40 \mathrm{sec}$. This US, in combination with DOX, did not increase the cytotoxic effects of DOX compared with the DOX only group, as validated by other researchers (23). Additionally, treatment with unloaded MBs + US had no obvious cytotoxic effect on the Raji cells compared with the control group (Fig. 5). However, the complicated mechanism of US irradiation-mediated release of drug-loaded MBs has not been thoroughly understood. We preliminarily analyzed the possibility that this mechanism could probably be involved in the following factors. i) There is high affinity between rituximab and CD-20 antigens expressed on the surface of cells. The specific conjugation between rituximab and CD-20 antigens contributes to the attachment of RDMs to Raji cells, improving the local chemotherapeutic accumulation (24). ii) US-mediated RDM oscillation and collapse contributes to the increased release of the encapsulated drug $(25,26)$. RDMs are more likely to preferentially accumulate and penetrate into cells through the EPR effect to promote the intracellular aggregation and uptake of drugs. iii) US-mediated RDM launch a powerful transmembrane shear force which facilitates the enhanced permeability of the extracellular drugs through the cell membrane (27). Pore formation, as well as endocytosis, have been reported during ultrasound application. Pore formation was shown to be caused by microbubbles propelled toward the cells (28). iv) Finally, the stimulation of US-mediated MBs can augment the influx of calcium ions in cells and increase the hydrogen peroxide level, factors that are closely associated with enhanced apoptosis (29). As confirmed by this set of experiments, the RDMs combined with US group revealed higher apoptosis efficiencies than the other treatment groups (Fig. 6). A parallel consequence was reported by other investigators (30).

Apoptosis is closely regulated through pro-apoptotic and anti-apoptotic factors. Based on the apoptosis factors evaluated in this study, the bcl-2 family is useful for evaluating apoptosis resulting from various types of stress (31). bcl-2 inhibits apoptosis by suppressing cytochrome $c$ release and impeding the activation of upstream caspase pathways (32). Translocation of the pro-apoptotic bax protein from the cytosol to the outer mitochondrial membrane results in cytochrome $c$ and Smac release via pore formation. Subsequently, cytochrome $c$ and caspase- 9 form apoptotic bodies through the apoptotic protease activating factor-1, and ultimately activate caspase-3, leading to apoptosis (33). Previous studies have confirmed that the levels of bcl-2 and bax protein expression relate directly to apoptotic regulation: bax elevation accelerates apoptosis and bcl-2 reduction promotes apoptosis. Therefore, the viability of cells after apoptosis induction is related to the ratio of bax to bcl-2. The results of the western blot analysis demonstrated that the level of bcl-2 protein was downregulated following treatment with RDMs + US, compared with the other treatment groups, whereas the bax protein was upregulated and the ratio of bax to bcl- 2 was elevated. In addition, the levels of active caspase- 3 proteins were significantly elevated in the RDMs + US group compared with other treatment groups. In short, these results revealed that RDMs + US can induce cell 
apoptosis through the bcl-2 protein family signaling pathway. The MB cavitation effect and the observation that the loaded drug was entirely released into the Raji cells may explain the mechanism of these cellular consequences.

In conclusion, the present study demonstrates the successful preparation of RDMs and US-mediated RDM release of DOX into Raji B cells. Our findings revealed that US-mediated RDMs can induce selective cytotoxicity and an apoptotic effect on Raji cells in vitro. Consequently, US-mediated RDM therapy, as described in this study, could be used as a novel and promising strategy for B cell NHL therapy.

\section{Acknowledgements}

The authors are grateful to the National Nature Science Foundation of P.R.China for its financial support (6590000065).

\section{References}

1. Witzig TE, Nowakowski GS, Habermann TM, Goy A, Hernandez-Ilizaliturri FJ, Chiappella A, Vitolo U, Fowler N and Czuczman MS: A comprehensive review of lenalidomide therapy for B-cell non-Hodgkin lymphoma. Ann Oncol 26: 1667-1677, 2015.

2. Feugier P, Van Hoof A, Sebban C, Solal-Celigny P, Bouabdallah R, Fermé C, Christian B, Lepage E, Tilly H, Morschhauser F, et al: Long-term results of the R-CHOP study in the treatment of elderly patients with diffuse large B-cell lymphoma: A study by the Groupe d'Etude des Lymphomes de l'Adulte. J Clin Oncol 23: 4117-4126, 2005.

3. Carvalho C, Santos RX, Cardoso S, Correia S, Oliveira PJ, Santos MS and Moreira PI: Doxorubicin: The good, the bad and the ugly effect. Curr Med Chem 16: 3267-3285, 2009.

4. Wheatley MA, Forsberg F, Oum K, Ro R and El-Sherif D: Comparison of in vitro and in vivo acoustic response of a novel 50:50 PLGA contrast agent. Ultrasonics 44: 360-367, 2006.

5. Xu JS, Huang J, Qin R, Hinkle GH, Povoski SP, Martin EW and $\mathrm{Xu}$ RX: Synthesizing and binding dual-mode poly (lactic-co-glycolic acid) (PLGA) nanobubbles for cancer targeting and imaging. Biomaterials 31: 1716-1722, 2010.

6. Acharya S and Sahoo SK: PLGA nanoparticles containing various anticancer agents and tumour delivery by EPR effect. Adv Drug Deliv Rev 63: 170-183, 2011.

7. Fan CH, Ting CY, Liu HL, Huang CY, Hsieh HY, Yen TC, Wei $\mathrm{KC}$ and Yeh CK: Antiangiogenic-targeting drug-loaded microbubbles combined with focused ultrasound for glioma treatment. Biomaterials 34: 2142-2155, 2013.

8. Zhang X, Zheng Y, Wang Z, Huang S, Chen Y, Jiang W, Zhang H, Ding M, Li Q, Xiao X, et al: Methotrexate-loaded PLGA nanobubbles for ultrasound imaging and synergistic targeted therapy of residual tumor during HIFU ablation. Biomaterials 35: $5148-5161,2014$.

9. Wang L, Li L, Guo Y, Tong H, Fan X, Ding J and Huang H: Construction and in vitro/in vivo targeting of PSMA-targeted nanoscale microbubbles in prostate cancer. Prostate 73 : 1147-1158, 2013.

10. Villa R, Cerroni B, Viganò L, Margheritelli S, Abolafio G, Oddo L, Paradossi G and Zaffaroni N: Targeted doxorubicin delivery by chitosan-galactosylated modified polymer microbubbles to hepatocarcinoma cells. Colloids Surf B Biointerfaces 110: 434-442, 2013.

11. Bao W, Liu R, Wang Y, Wang F, Xia G, Zhang H, Li X, Yin H and Chen B: PLGA-PLL-PEG-Tf-based targeted nanoparticles drug delivery system enhance antitumor efficacy via intrinsic apoptosis pathway. Int J Nanomedicine 10: 557-566, 2015.

12. Liu R, Wang Y, Li X, Bao W, Xia G, Chen W, Cheng J, Xu Y, Guo L and Chen B: Synthesis and characterization of tumor-targeted copolymer nanocarrier modified by transferrin. Drug Des Devel Ther 9: 2705-2719, 2015.

13. Geers B, De Wever O, Demeester J, Bracke M, De Smedt SC and Lentacker I: Targeted liposome-loaded microbubbles for cell-specific ultrasound-triggered drug delivery. Small 9: 4027-4035, 2013.
14. Liu H, Chang S, Sun J, Zhu S, Pu C, Zhu Y, Wang Z and Xu RX: Ultrasound-mediated destruction of LHRHa-targeted and paclitaxel-loaded lipid microbubbles induces proliferation inhibition and apoptosis in ovarian cancer cells. Mol Pharm 11: 40-48, 2014.

15. Wang J, Wang M,Zheng M, Guo Q, Wang Y, Wang H, Xie X, Huang F and Gong R: Folate mediated self-assembled phytosterol-alginate nanoparticles for targeted intracellular anticancer drug delivery. Colloids Surf B Biointerfaces 129: 63-70, 2015.

16. Lee SJ, Shim YH, Oh JS, Jeong YI, Park IK and Lee HC: Folic-acid-conjugated pullulan/poly(DL-lactide-co-glycolide) graft copolymer nanoparticles for folate-receptor-mediated drug delivery. Nanoscale Res Lett 10: 43, 2015.

17. Niu C, Wang Z, Lu G, Krupka TM, Sun Y, You Y, Song W, Ran H, Li P and Zheng Y: Doxorubicin loaded superparamagnetic PLGA-iron oxide multifunctional microbubbles for dual-mode US/MR imaging and therapy of metastasis in lymph nodes. Biomaterials 34: 2307-2317, 2013.

18. Feugier P: A review of rituximab, the first anti-CD20 monoclonal antibody used in the treatment of B non-Hodgkin's lymphomas. Future Oncol 11: 1327-1342, 2015.

19. Zheng Y, Zhang Y, Ao M, Zhang P, Zhang H, Li P, Qing L, Wang Z and Ran H: Hematoporphyrin encapsulated PLGA microbubble for contrast enhanced ultrasound imaging and sonodynamic therapy. J Microencapsul 29: 437-444, 2012.

20. Kang J, Wu X, Wang Z, Ran H, Xu C, Wu J, Wang Z and Zhang Y: Antitumor effect of docetaxel-loaded lipid microbubbles combined with ultrasound-targeted microbubble activation on VX2 rabbit liver tumors. J Ultrasound Med 29: 61-70, 2010

21. Pu C, Chang S, Sun J, Zhu S, Liu H, Zhu Y, Wang Z and Xu RX: Ultrasound-mediated destruction of LHRHa-targeted and paclitaxel-loaded lipid microbubbles for the treatment of intraperitoneal ovarian cancer xenografts. Mol Pharm 11: 49-58, 2014.

22. Veiseh O, Gunn JW and Zhang M: Design and fabrication of magnetic nanoparticles for targeted drug delivery and imaging. Adv Drug Deliv Rev 62: 284-304, 2010.

23. Tinkov S, Winter G, Coester C and Bekeredjian R: New doxorubicin-loaded phospholipid microbubbles for targeted tumor therapy: Part I - Formulation development and in-vitro characterization. J Control Release 143: 143-150, 2010.

24. Yan F, Li X, Jin Q, Jiang C, Zhang Z, Ling T, Qiu B and Zheng H: Therapeutic ultrasonic microbubbles carrying paclitaxel and LyP-1 peptide: Preparation, characterization and application to ultrasound-assisted chemotherapy in breast cancer cells. Ultrasound Med Biol 37: 768-779, 2011.

25. Escoffre JM, Novell A, Piron J, Zeghimi A, Doinikov A and Bouakaz A: Microbubble attenuation and destruction: Are they involved in sonoporation efficiency? IEEE Trans Ultrason Ferroelectr Freq Control 60: 46-52, 2013

26. Luan Y, Faez T, Gelderblom E, Skachkov I, Geers B, Lentacker I, van der Steen T, Versluis M and de Jong N: Acoustical properties of individual liposome-loaded microbubbles. Ultrasound Med Biol 38: 2174-2185, 2012.

27. Geers B, Lentacker I, Alonso A, Sanders NN, Demeester J, Meairs S and De Smedt SC: Elucidating the mechanisms behind sonoporation with adeno-associated virus-loaded microbubbles. Mol Pharm 8: 2244-2251, 2011.

28. De Cock I, Zagato E, Braeckmans K, Luan Y, de Jong N, De Smedt SC and Lentacker I: Ultrasound and microbubble mediated drug delivery: Acoustic pressure as determinant for uptake via membrane pores or endocytosis. J Control Release 197: 20-28, 2015.

29. Juffermans LJ, Meijering DB, van Wamel A, Henning RH, Kooiman K, Emmer M, de Jong N, van Gilst WH, Musters R, Paulus WJ, et al: Ultrasound and microbubble-targeted delivery of therapeutic compounds: ICIN Report Project 49: Drug and gene delivery through ultrasound and microbubbles. Neth Heart J 17: 82-86, 2009.

30. Yang S, Wang P, Wang X, Su X and Liu Q: Activation of microbubbles by low-level therapeutic ultrasound enhances the antitumor effects of doxorubicin. Eur Radiol 24: 2739-2753, 2014

31. Babu PP, Suzuki G, Ono Y and Yoshida Y: Attenuation of ischemia and/or reperfusion injury during myocardial infarction using mild hypothermia in rats: An immunohistochemical study of Bcl-2, Bax, Bak and TUNEL. Pathol Int 54: 896-903, 2004.

32. Voutsadakis IA: Apoptosis and the pathogenesis of lymphoma. Acta Oncol 39: 151-156, 2000.

33. Allen TM: Ligand-targeted therapeutics in anticancer therapy. Nat Rev Cancer 2: 750-763, 2002. 